\title{
A EFICIÊNCIA TÉCNICA NOS INVESTIMENTOS NO SETOR DE SAÚDE: UM ESTUDO NA REGIÃO METROPOLITANA DO VALE DO PARAÍBA E LITORAL NORTE DE SÃO PAULO
}

\author{
THE TECHNICAL EFFICIENCY IN INVESTMENTS IN THE HEALTH \\ SECTOR: A STUDY IN THE METROPOLITAN REGION OF THE \\ PARAÍBA VALLEY AND NORTH COAST OF SÃO PAULO
}

\begin{abstract}
Andréa Aparecida Ferreira Silva ${ }^{1}$ Marlette Cassia Oliveira Ferreira ${ }^{2}$ Jussara da Silva Teixeira Cucato ${ }^{3}$ Jussara Goulart da Silva
\end{abstract}

\begin{abstract}
Resumo: O presente estudo tem como objetivo investigar a eficiência técnica da alocação de recursos públicos nos serviços de saúde, tomando como referência a Região Metropolitana do Vale do Paraíba e Litoral Norte de São Paulo. Para avaliar a eficiência técnica do setor de saúde foram selecionadas variáveis que representaram os recursos alocados (inputs) e os serviços oferecidos à sociedade (outputs). A mensuração da eficiência foi realizada por intermédio da Análise Envoltória de Dados, com a utilização do modelo clássico de retornos variáveis com orientação produto. A fronteira de eficiência obtida não busca fornecer um padrão rígido de eficiência para a saúde, mas uma forma de verificar o quanto a região poderia produzir se mantido o atual investimento. Os resultados demonstraram que 50\% dos municípios analisados apresentaram eficiência técnica relativa de $100 \%$ e o índice médio alcançado na região foi de $87,7 \%$. A pesquisa evidenciou, ainda, que para uma cidade da amostra alcançar a fronteira de eficiência técnica não precisa necessariamente realizar maiores investimentos.
\end{abstract}

Palavras-chave: Saúde. Investimento. DEA. Eficiência.

Abstract: This study aims to investigate the technical efficiency of the allocation of public resources in health services, taking as reference the Metropolitan Region of Vale do Paraíba and the North Coast of São Paulo. To assess the technical efficiency of the health sector, variables that represent the allocated resources (inputs) and the services offered to society (outputs) were selected. The measurement of efficiency was performed through the Data Envelopment Analysis, using the classic model of variable returns with product orientation. The efficiency frontier obtained does not seek to provide a rigid standard of efficiency for health, but a way of verifying how much the region could produce if the current investment is maintained. The results showed that $50 \%$ of the municipalities analyzed showed relative technical efficiency of $100 \%$ and the average index reached in the region was $87.7 \%$. The research also showed that for a city in the sample to reach the frontier of technical efficiency, it does not necessarily need to make greater investments.

Keywords: Health. Investment. DEA. Efficiency.

\footnotetext{
(D) ${ }^{1}$ Especialista em Gestão Financeira. Instituto Federal de São Paulo - IFSP. Caraguatatuba, São Paulo. Brasil. andrea.etec@gmail.com

(D) 2 Doutora em Administração. Instituto Federal de São Paulo - IFSP. Caraguatatuba, São Paulo. Brasil. marlettecassia@ gmail.com

(D) ${ }^{3}$ Mestre em Administração. Escola Superior de Propaganda e Marketing - ESPM. São Paulo, SP. Brasil. jussaracucato@gmail.com

(D) ${ }^{4}$ Doutora em Administração. Universidade Federal de Uberlândia. Uberlândia, Minas Gerais. Brasil. profadmjussara.ufu@gmail.com
}

\section{Cite como}

American Psychological Association (APA)

Silva, A. A. F., Ferreira, M. C. O., Cucato, J. S. T., \& Silva, J. G. (2020, jul./dez.). A eficiência técnica nos investimentos no setor de saúde: um estudo na região metropolitana do Vale do Paraíba e litoral norte de São Paulo . Revista Inovação, Projetos e Tecnologias, São Paulo, 8(2), 270-285. https://doi.org/10.5585/iptec.v8i2.18783. 


\section{Introdução}

A utilização de técnicas e métodos de avaliação de políticas públicas de saúde é indispensável para equacionar o setor, de forma que tanto os dirigentes do governo quanto os pesquisadores da área de gestão e da área de saúde estão empenhados em investigar a qualidade e a eficiência da prestação dos serviços de saúde pública no Brasil (Hartz \& Pouvourville, 1998; Fonseca \& Ferreira, 2009).

As três esferas de governo - Federal, Estadual e Municipal - objetivam a racionalidade na utilização dos recursos públicos, destarte reitera-se a premência de pesquisas que permitam indicar a destinação das verbas públicas de forma eficiente. $\mathrm{O}$ aperfeiçoamento das técnicas e dos instrumentos de avaliação pode contribuir para a tomada de decisão nas políticas públicas do país e, com isso, promover o alcance de mais eficiência e melhores resultados com os gastos públicos (Marinho \& Façanha, 2001; Costa \& Castanhar, 2003).

A descentralização das ações e dos serviços de saúde, a partir da Constituição de 1988, tornou a administração dos recursos financeiros transferidos do Ministério da Saúde para os Fundos Municipais de Saúde uma responsabilidade do gestor municipal. Grande parte das ações de saúde municipais é decidida em nível microrregional, dada a atuação em rede promovida pela descentralização, em que um ou mais municípios são tidos como referência para a microrregião (Hartz \& Pouvourville, 1998; Fonseca \& Ferreira, 2009).

Com foco nesse tipo de controle, este estudo propõe-se a responder à seguinte pergunta:

Qual o nível de eficiência técnica dos municípios da Região Metropolitana do Vale do Paraíba e Litoral Norte de São Paulo na alocação de recursos públicos nos serviços de saúde, no ano de 2014?

Operar o sistema com eficiência, ou seja, transformar os insumos sejam eles tecnológicos, humanos ou de capital, no máximo de produtos e serviços possíveis, respeitando os parâmetros técnicos, os recursos disponíveis e as especificidades regionais, é de interesse de toda a sociedade e dever dos gestores, em razão de restrições orçamentárias (Costa \& Castanhar, 2003).

Apesar de organizações públicas de saúde já utilizarem indicadores como o Índice de Desenvolvimento Humano (IDH) e coeficientes epidemiológicos e de morbidade, entre outros, para o controle da situação da saúde, ainda são escassos indicadores de eficiência relativa e programação linear para comparar regiões distintas. Tamanha a necessidade de mensurar a eficiência dos serviços prestados, que o Tribunal de Contas do Estado de São Paulo (TCE-SP) criou, em 2015, o Índice de Efetividade da Gestão Municipal (IEG-M), para medir os resultados 
dos municípios (Costa \& Castanhar, 2003; Tribunal de Contas do Estado de São Paulo [TCESP], 2017).

Portanto, em face da limitação dos recursos disponíveis e da falta de acesso aos serviços básicos de saúde, é oportuno mensurar a eficiência dos investimentos realizados pelos municípios. Os dados disponibilizados para o Ministério da Saúde podem ser uma alternativa de controle e monitoramento para buscar benchmark na intenção de aumentar a oferta de atendimento aos cidadãos (Grassetti, Gori, \& Bellio, 2003; Machado Junior, Irffi, \& Benegas, 2011).

Pesquisadores da área de gestão, da saúde e do poder executivo empenham-se em avaliar a qualidade e a eficiência da prestação dos serviços de saúde pública. Nesse sentido, cabe citar os trabalhos de Marinho e Façanha (2001), Grassetti et al. (2003), Faria, Jannuzzi e Silva (2008), Fonseca e Ferreira (2009), Silva (2010), Machado Junior et al. (2011), Oliveira (2012), Passoni (2014), Queiroz, Silva, Figueiredo e Vale (2013), demonstrando a necessidade da utilização de tecnologias que permitam avaliações no setor, tais como métodos de controle das políticas públicas de saúde.

Buscando contribuir com essa discussão, o presente estudo fará uso da técnica de análise de dados Data Envelopment Analysis (DEA), mais conhecida no Brasil por Análise Envoltória dos Dados, que visa quantificar e comparar a eficiência na utilização de recursos para o provimento de bens e serviços.

\section{Revisão da literatura}

O aperfeiçoamento de instrumentos e técnicas para tomada de decisão e para avaliação das políticas públicas no país tem dirigido a necessidade de se obter melhor aproveitamento dos recursos públicos. Atender aos acréscimos de demanda social por serviços de saúde e do financiamento por mais impostos sem ponderar as maneiras como esses recursos são explorados em países pobres como o Brasil, onde a mensuração da eficiência no setor saúde é fundamental (Marinho \& Façanha, 2001; Costa \& Castanhar, 2003; Fonseca \& Ferreira, 2009; Passoni, 2014).

A metodologia Data Envelopment Analysis (DEA), conhecida no Brasil como Análise Envoltória de Dados, é uma ferramenta utilizada pelos pesquisadores quando se tem por objetivo avaliar, mensurar e comparar a eficiência produtiva de unidades organizacionais homogêneas, tais como: hospitais, escolas, indústrias, entre outras. A DEA vem sendo bastante 
utilizada na análise da eficiência dos gastos públicos, comparando as despesas públicas dos municípios e estados (Machado Junior et al., 2011; Dalchiavon \& Melo, 2016).

O trabalho pioneiro em análise da eficiência foi realizado em 1957, por Farrell, utilizando programação matemática para aferir a eficiência técnica em casos de único produto/insumo. A utilização dessa técnica para a análise da eficiência dos gastos públicos surgiu com o trabalho de Charnes, Cooper e Rhodes (1978), no qual buscou comparar a eficiência de escolas públicas. Na literatura contemporânea encontram-se diversas pesquisas que empregam a metodologia $D E A$, tanto internacionais como nacionais, e com diversos intuitos.

Marinho (2003) implementou uma análise da eficiência técnica nos serviços de saúde no ano de 1996, nos municípios do Rio de Janeiro. O autor utilizou o modelo DEA e a regressão múltipla para avaliar os serviços ambulatoriais e hospitalares. Observou-se, com base nos resultados, que os níveis de eficiência gerais dos municípios cariocas são medianos e que a concentração da população residente nas áreas eficientes é menor que $20 \%$. Verificou-se, ainda, que o centro sul do estado está à frente do norte-nordeste, contudo, as peculiaridades regionais não são muito significativas.

Dias (2010) estudou a eficiência da Atenção Primária à Saúde nos municípios brasileiros, no ano de 2006. O autor utilizou, além da técnica de Análise Envoltória dos Dados, a regressão múltipla, visando investigar não só a eficiência na prestação de serviços de saúde da atenção básica como também a interferência de elementos externos nessa prestação.

Machado Junior et al. (2011) utilizaram dados do Instituto de Pesquisa e Estratégia Econômica do Ceará (IPECE), Ministério da Fazenda e Secretaria do Tesouro Nacional (STN), IPEADATA, Instituto Nacional de Estudos e Pesquisas Educacionais Anísio Teixeira (INEP), para investigar a eficiência técnica das despesas públicas municipais per capita em educação, saúde e assistência social do ano de 2005, no Ceará. Os resultados mostraram que, quanto ao modelo de despesa pública, 55\% dos municípios cearenses ficaram sobre a fronteira de eficiência relativa, todavia, existe reduzida eficiência técnica na despesa pública social no Estado.

Schulz, Gollo, Rosa e Scarpin (2014) verificaram o desempenho das unidades federativas brasileiras, por meio da técnica $D E A$, em relação a seus investimentos públicos em saúde no período de 2005 a 2011. A pesquisa indicou que oito unidades federativas foram $100 \%$ eficientes em pelo menos um dos períodos analisados, com relação a estabelecimentos de saúde, internações, pessoas cadastradas na atenção básica, produção ambulatorial, equipamentos e imunizações. 
Tomando-se por base os estudos relacionados, observa-se que a questão da eficiência dos serviços públicos de saúde tem ocupado elevado espaço nas agendas de pesquisa, tanto na literatura internacional quanto nacional, evidenciando sua importância na avaliação da gestão dos gastos públicos com saúde (Nunes \& Sousa, 2016).

\section{Metodologia}

Com o intuito de responder ao objetivo geral proposto nesta pesquisa, foi constituída uma pesquisa de natureza básica. Realizou-se a análise teórica relativa aos fatos com o propósito de originar informações novas e úteis, tanto para administração dos recursos públicos como para difundir o conhecimento dos investimentos realizados na saúde dos municípios estudados (Shaughnessy, Zechmeister, \& Zechmeister, 2012; Cozby, 2014).

O procedimento metodológico contemplado no estudo refere-se à Análise Envoltória de Dados (DEA - Data Envelopment Analysis), buscando determinar os scores de eficiência técnica dos gastos públicos com saúde nos municípios selecionados. O método possibilita a determinação da eficiência de uma unidade produtiva, denominadas DMUs (Decision Making Units). Trata-se de um instrumento não paramétrico que avalia a eficiência de $D M U s$ e é considerado uma ferramenta matemática, realizando um comparativo das unidades que fazem atividades semelhantes e se distinguem pelo montante de recursos usados (inputs) e de produtos gerados (outputs) (Farrel, 1957; Charnes et al., 1978).

O emprego de DEA permite reconhecer benchmarks, ou seja, referências baseadas no grau de performance relativo de unidades semelhantes, que utilizam insumos (inputs) para gerar produtos (outputs). O modelo constrói a fronteira de eficiência pautado na melhor relação insumo/produto. As unidades menos eficientes ficam estabelecidas numa área inferior à fronteira, conhecida como envelope (envoltória) e para cada unidade nesta posição a $D E A$ fornece um índice de desempenho abaixo de $100 \%$ e reconhece um subconjunto de unidades posicionadas na fronteira para lhe servirem de referência (Machado Junior et al., 2011; Charnes et al., 1978).

Neste estudo, utilizou-se o modelo $D E A B C C$ que considera retornos variáveis de escala, também denominado Variable Returns to Scale (VRS), com orientação para maximização de output, quando o nível é calculado por meio da maximização de produtos, dada uma quantidade de insumos utilizados (Banker, Charnes, \& Cooper, 1984), considerando-se as restrições orçamentárias do setor de saúde. 
A abordagem é principalmente quantitativa (Malhotra, 2012), com amostragem não probabilística (Hair Júnior et al., 2005), sendo a amostra selecionada por conveniência e os dados foram trabalhados em termos numéricos e percentuais (Gil, 2010).

Foram utilizados métodos descritivos (Gil, 2010; Andrade, 2010) na observação dos dados informados pelas prefeituras ao Ministério da Saúde. A população foi composta pelas 39 cidades da Região Metropolitana do Vale do Paraíba e Litoral Norte do estado de São Paulo, referente ao ano base disponível para variável 'Famílias Cadastradas'. A amostra foi determinada pelos municípios que disponibilizaram informações sobre saúde pública, sendo eliminadas as cidades com informações incompletas: Cruzeiro; Lavrinhas; Natividade da Serra; Pindamonhangaba; Potim; Roseira; Santa Branca; São Bento do Sapucaí; São José do Barreiro.

\subsection{Seleção das variáveis e coleta dos dados}

As variáveis utilizadas no modelo $D E A$ foram selecionadas considerando-se as evidências empíricas encontradas em outras pesquisas e que se mostraram consistentes na definição da eficiência técnica (Queiroz et al., 2013; Schulz et al., 2014; Duarte, Diniz, Nobre, \& Araújo, 2016), conforme expressa o Quadro 1.

Quadro 1 - Variáveis pesquisadas

\begin{tabular}{|c|c|c|c|}
\hline Tipo & Variável & Descrição & Fonte \\
\hline Input_l & $\begin{array}{l}\text { Despesa Total em } \\
\text { Saúde }\end{array}$ & Total da Despesa com a função Saúde. & $\begin{array}{l}\text { SIOPS } \\
(2014)\end{array}$ \\
\hline Output_1 & $\begin{array}{l}\text { Produção } \\
\text { Ambulatorial do } \\
\text { SUS }\end{array}$ & $\begin{array}{l}\text { Quantidade Aprovada de Produção Ambulatorial } \\
\text { realizada pelo SUS. }\end{array}$ & \multirow{4}{*}{$\begin{array}{l}\text { DATASUS } \\
(2014)\end{array}$} \\
\hline Output_2 & Imunizações & $\begin{array}{l}\text { Quantidade de doses aplicadas em ações de vacinação } \\
\text { por município considerando população-alvo }\end{array}$ & \\
\hline Output_3 & Número de Visitas & $\begin{array}{l}\text { Número de Visitas às Famílias das equipes de Saúde da } \\
\text { Família (eSF) e Agentes Comunitários de Saúde (ACS). }\end{array}$ & \\
\hline Output_4 & $\begin{array}{l}\text { Famílias } \\
\text { Cadastradas }\end{array}$ & $\begin{array}{l}\text { Quantidade de famílias cadastradas nas equipes de Saúde } \\
\text { da Família. }\end{array}$ & \\
\hline
\end{tabular}

Fonte: Dados da pesquisa (2018).

A Despesa Total representa os gastos totais que cada município destinou para a função saúde pública no ano de 2014, informados ao Ministério da Saúde por meio do SIOPS (2014). A Produção Ambulatorial do SUS condiz com a quantidade de procedimentos aprovados pelas 
Secretarias de Saúde e informados no Sistema de Informações Ambulatoriais (SIA/SUS); a variável Imunizações equivale à quantidade de doses de vacinas aplicadas à população-alvo, cujos dados são disponibilizados por meio do Sistema de Avaliação do Programa de Imunizações (API); o Número de Visitas refere-se aos dados alcançados pelo trabalho das equipes de Saúde da Família (eSF) e Agentes Comunitários de Saúde (ACS) provenientes do Sistema de Informação da Atenção Básica (SIAB); e as Famílias Cadastradas equivale à quantidade de famílias que foram cadastradas nas eSF, também no SIAB. Todos os dados estão disponíveis no site do Departamento de Informática do Sistema Único de Saúde do Brasil, Datasus (2014).

Por fim, foram elencados os valores per capita investidos em saúde por cada município da amostra, com o objetivo de comparar os volumes aplicados com os níveis de eficiência obtidos. Os dados foram retirados do site IBGE Cidades e são referentes ao último censo, realizado no ano de 2010.

\subsection{Tratamento dos dados}

Preliminarmente, realizou-se uma caracterização das variáveis utilizadas no modelo, destacando as estatísticas descritivas, os valores máximos e mínimos observados na amostra. Em seguida, utilizou-se software para auxiliar no cálculo da eficiência das DMUs, empregando o modelo DEA. O software escolhido foi o Sistema Integrado de Apoio à Decisão (SIAD) versão 3.0, desenvolvido por Angulo-Meza, Biondi Neto, Mello e Gomes (2005) na Universidade Federal Fluminense, por ser um programa computacional distribuído de forma gratuita. Calculou-se os scores de eficiência padrão dos municípios, os benchmarks de cada unidade ineficiente e na sequência os valores dos produtos que seriam necessários para tornálos eficientes. Por último, comparou-se os gastos per capita com os scores de eficiência alcançados, utilizando-se o programa licenciado Microsoft Excel versão Office $2010^{\circledR}$ para manejo dos dados.

\section{Apresentação dos resultados}

O estudo empregou como input a Despesa Total que cada município destinou na função saúde no exercício de 2014. E, como outputs, as variáveis adotadas referem-se aos resultados atingidos por cada cidade em cada variável. A Tabela 1 apresenta as estatísticas descritivas.

Os dados da Tabela 1 permitem verificar que a média das Despesas Totais em Saúde no ano de 2014 ultrapassou 42,3 milhões de reais. Entretanto, o desvio padrão desta variável 
evidencia a variabilidade dos gastos dos municípios com saúde em torno da média, destacando o investimento máximo de mais de 519 milhões de reais e o valor mínimo de pouco mais de 2,5 milhões de reais.

Tabela 1 - Estatísticas descritivas das variáveis

\begin{tabular}{|c|c|c|c|c|c|c|}
\hline Variáveis & Tipo & Total & Média & $\begin{array}{l}\text { Desvio } \\
\text { Padrão }\end{array}$ & Máximo & Mínimo \\
\hline $\begin{array}{l}\text { Despesa Total } \\
\text { em Saúde }\end{array}$ & Insumo & $\begin{array}{l}\mathrm{R} \$ \\
1.652 .229 .470,02\end{array}$ & $\begin{array}{l}\mathrm{R} \$ \\
42.364 .858,21\end{array}$ & $\begin{array}{l}\mathrm{R} \$ \\
88.280 .127,84\end{array}$ & $\begin{array}{l}\mathrm{R} \$ \\
519.271 .605,02\end{array}$ & $\begin{array}{l}\mathrm{R} \$ \\
2.545 .303,09\end{array}$ \\
\hline Produção & & & & & & \\
\hline $\begin{array}{l}\text { Ambulatorial do } \\
\text { SUS }\end{array}$ & Produto & 57.102 .940 & 1.464 .178 & 3.145 .980 & 16.817 .794 & 54.391 \\
\hline Imunizações & Produto & 1.922 .41 & 49.293 & 97.623 & 542.721 & 1.605 \\
\hline $\begin{array}{l}\text { Número de } \\
\text { Visitas }\end{array}$ & Produto & 321.225 & 8.682 & 7.391 & 34.439 & 1.165 \\
\hline $\begin{array}{l}\text { Famílias } \\
\text { Cadastradas }\end{array}$ & Produto & 239.847 & 7.995 & 8.133 & 27.729 & 189 \\
\hline
\end{tabular}

Fonte: Dados da pesquisa (2018).

Tal comportamento em relação à média pode ser observado também nas demais variáveis, enfatizando as desigualdades existentes entre as cidades da amostra. Apesar disso, é importante ressaltar que essa variabilidade pode ser justificada pelo tamanho dos municípios.

\subsection{Scores da eficiência técnica - modelo DEA-BCC}

A análise da eficiência padrão associa o valor de $100 \%$ às $D M U s$ mais eficientes, no caso os municípios selecionados. A eficiência é entendida por Farrel (1957) como a relação ótima entre os insumos e métodos necessários (inputs) e produtos ou serviços (outputs) no processo. Dessa forma, a eficiência no âmbito da saúde pública incide na destinação mais acertada dos recursos públicos para oferecer ao cidadão a maior quantidade possível de produtos e serviços.

O princípio básico de utilização da $D E A$ pode ser definido conforme apresentado por Fonseca e Ferreira (2009) e corroborado por Machado Junior et al. (2011), onde uma DMU é considerada eficiente quando produz a maior quantidade de produtos (outputs) usando uma determinada quantidade de insumos (inputs) e as demais DMUs serão eficientes se capazes de produzir o mesmo resultado. O DEA faz a combinação de todas as $D M U s$ avaliadas como 
eficientes e forma uma $D M U$ virtual com inputs e outputs virtuais. Quando uma $D M U$ real não consegue produzir mais que a $D M U$ virtual usando a mesma quantidade de insumos, ou, a $D M U$ real não consegue obter a mesma produção que a $D M U$ virtual com uma quantidade menor de insumos, então essa $D M U$ real é analisada como ineficiente.

Desta forma, os resultados obtidos pelas cidades de Arapeí, Areias, Caçapava, Cachoeira Paulista, Canas, Caraguatatuba, Jacareí, Lorena, Monteiro Lobato, Piquete, São José dos Campos, São Sebastião, Taubaté, Tremembé e Ubatuba, em relação às demais cidades, classificaram-nas como municípios eficientes na aplicação dos recursos públicos em saúde. As demais cidades ficaram abaixo da fronteira da eficiência, como mostra a Tabela 2.

Tabela 2 - Scores dos municípios abaixo da fronteira de eficiência técnica

$\begin{array}{llllll}\text { DMU } & \text { Score } & \text { DMU } & \text { Score } & \text { DMU } & \text { Score } \\ \text { São Luiz do Paraitinga } & 99,01 \% & \text { Paraibuna } & 80,16 \% & \text { Lagoinha } & 65,24 \% \\ \text { Campos do Jordão } & 98,86 \% & \text { Guaratinguetá } & 77,51 \% & \text { Cunha } & 64,65 \% \\ \text { Aparecida } & 96,68 \% & \text { Redenção da } & 71,10 \% & \text { Ilhabela } & 56,06 \% \\ \text { Igaratá } & & \text { Serra } & & & 54,31 \% \\ \text { Santo Antônio do Pinhal } & 90,36 \% & \text { Silveiras } & 65,50 \% & \text { Jambeiro } & 49,27 \%\end{array}$

Fonte: Dados da pesquisa (2018).

A amostra demonstrou-se predominantemente eficiente, com 20 dos 30 municípios avaliados acima da média dos scores de eficiência técnica obtido, 87,70\%. Pode-se verificar que, de forma geral, as cidades estudadas foram eficientes e possuem aptidão por fazer o uso mais adequado daquilo que se tem à disposição para obter o resultado pretendido.

\subsection{Benchmark para as DMUs ineficientes}

Um dos benefícios do uso da $D E A$ é o grupo de unidades de referência que pode ser usado como benchmark na melhoria do desempenho das unidades menos eficientes. Estes benchmarks indicam as modificações necessárias para transformar unidades ineficientes em eficientes. O benchmark pode ser determinado como processo sistemático e contínuo para identificar a melhor prática e consequentemente a modificação do conhecimento existente, de modo a conseguir uma atuação superior.

As cidades com scores de eficiência de $100 \%$ são identificadas pelo software como benchmark para os municípios que ficaram aquém da fronteira da eficiência técnica. $\mathrm{O}$ 
município de Canas foi identificado como benchmark para as cidades de Igaratá, Jambeiro, Lagoinha, Redenção da Serra e Silveiras; Tremembé foi benchmark para Aparecida, Campos do Jordão, Cunha e Guaratinguetá; Piquete mostrou-se benchmark para Queluz, Santo Antônio do Pinhal e São Luiz do Paraitinga; para Bananal e Paraibuna o benchmark é Cachoeira Paulista; e Ubatuba apresentou-se como benchmark para Ilhabela.

Os dados desse estudo convergem com os pensamentos de Grassetti et al. (2003) confirmados posteriormente por Machado et al. (2011), uma vez que analisar a eficiência viabiliza o estabelecimento de metas de desempenho díspares e torna-se útil aos gestores por ser capaz de indicar o potencial de melhoria na atuação de cada setor e ainda a definição de indicadores relativos às referências de cada organização permitindo, assim, a análise diferenciada de seu funcionamento.

Os resultados da $D E A$ permitiram arrazoar sobre o que seria necessário para esses municípios se localizarem sobre a fronteira de eficiência técnica relativa, ou seja, tornem-se eficientes na aplicação dos recursos públicos em serviços de saúde. A Tabela 3 apresenta as variáveis com os valores percentuais indicados pela Data Envelopment Analysis para melhoraria do desempenho das $D M U s$ menos eficientes, mantendo o mesmo valor investido, destacando-se os três municípios com os piores resultados da amostra, de acordo com os resultados da pesquisa. 
Tabela 3 - Valores percentuais indicados pela $D E A$ para alcançar a fronteira da eficiência

\begin{tabular}{|c|c|c|c|c|c|}
\hline Variável & $\begin{array}{l}\text { Despesa Total } \\
\text { Saúde }\end{array}$ & $\begin{array}{l}\text { Produção } \\
\text { Ambulatorial }\end{array}$ & Imunização & $\begin{array}{l}\text { Número de } \\
\text { Visitas }\end{array}$ & $\begin{array}{l}\text { Famílias } \\
\text { Cadastradas }\end{array}$ \\
\hline Aparecida & - & $3 \%$ & $3 \%$ & $3 \%$ & $3 \%$ \\
\hline Bananal & - & $84 \%$ & $92 \%$ & $84 \%$ & $84 \%$ \\
\hline Campos do Jordão & - & $144 \%$ & $1 \%$ & $1 \%$ & $1 \%$ \\
\hline Cunha & - & $55 \%$ & $55 \%$ & $55 \%$ & $2.280 \%$ \\
\hline Guaratinguetá & - & $29 \%$ & $29 \%$ & $521 \%$ & $29 \%$ \\
\hline Igaratá & - & $33 \%$ & $9 \%$ & $9 \%$ & $9 \%$ \\
\hline Ilhabela & - & $301 \%$ & $78 \%$ & $\mathbf{8 8 \%}$ & $78 \%$ \\
\hline Jambeiro & - & $103 \%$ & $103 \%$ & $103 \%$ & $103 \%$ \\
\hline Lagoinha & - & $53 \%$ & $112 \%$ & $53 \%$ & $53 \%$ \\
\hline Paraibuna & - & $44 \%$ & $25 \%$ & $73 \%$ & $25 \%$ \\
\hline Queluz & - & $42 \%$ & $42 \%$ & $42 \%$ & $42 \%$ \\
\hline Redenção da Serra & - & $41 \%$ & $62 \%$ & $41 \%$ & $65 \%$ \\
\hline Santo Antônio do Pinhal & - & $11 \%$ & $11 \%$ & $11 \%$ & $11 \%$ \\
\hline São Luiz do Paraitinga & - & $37 \%$ & $17 \%$ & $1 \%$ & $1 \%$ \\
\hline Silveiras & - & $53 \%$ & $66 \%$ & $53 \%$ & $53 \%$ \\
\hline
\end{tabular}

Fonte: Dados da pesquisa (2018)

\subsection{Relação scores da eficiência técnica versus investimento em saúde per capita}

Visando comparar os scores da eficiência técnica alcançados pelos municípios com o investimento realizado em saúde per capita, foram listados os valores alocados pelas cidades analisadas. A Tabela 4 apresenta o ranking dos recursos per capita investidos no ano de 2014, em que é possível observar que os scores de eficiência obtidos não acompanham o ranking. Existem cidades com os maiores valores per capita investidos, como é o caso de Ilhabela, que figuraram entre os piores índices de eficiência: 
Tabela 4 - Ranking dos recursos per capita investidos no ano de 2014

\begin{tabular}{|c|c|c|c|c|c|c|c|}
\hline & Município & $\begin{array}{l}\text { Despesa } \\
\text { per capita }\end{array}$ & $\begin{array}{l}\text { Score de } \\
\text { Eficiência }\end{array}$ & & Município & $\begin{array}{l}\text { Despesa } \\
\text { per capita }\end{array}$ & $\begin{array}{l}\text { Score de } \\
\text { Eficiência }\end{array}$ \\
\hline o & Ilhabela & $1.500,46$ & $56,06 \%$ & $6^{\circ}$ & $\begin{array}{l}\text { Redenção da } \\
\text { Serra }\end{array}$ & 683,12 & $71,10 \%$ \\
\hline o & São Sebastião & $1.491,20$ & $100 \%$ & $7^{\circ}$ & Jacareí & 671,76 & $100 \%$ \\
\hline o & Arapeí & $1.368,43$ & $100 \%$ & $8^{\circ}$ & Guaratinguetá & 670,48 & $77,51 \%$ \\
\hline o & Jambeiro & $1.223,60$ & $49,27 \%$ & $9^{\circ}$ & $\begin{array}{l}\text { Santo Antônio } \\
\text { do Pinhal }\end{array}$ & 643,19 & $90,36 \%$ \\
\hline o & Lagoinha & 997,23 & $65,24 \%$ & $0^{\circ}$ & Paraibuna & 634,22 & $80,16 \%$ \\
\hline o & $\begin{array}{l}\text { Monteiro } \\
\text { Lobato }\end{array}$ & 954,57 & $100 \%$ & $1^{\circ}$ & Lorena & 604,68 & $100 \%$ \\
\hline o & Caraguatatuba & 925,24 & $100 \%$ & $2^{\circ}$ & Queluz & 567,91 & $70,48 \%$ \\
\hline o & Areias & 838,12 & $100 \%$ & $3^{\circ}$ & Taubaté & 553,60 & $100 \%$ \\
\hline o & $\begin{array}{l}\text { São José dos } \\
\text { Campos }\end{array}$ & 824,34 & $100 \%$ & $4^{\circ}$ & $\begin{array}{l}\text { São Luiz do } \\
\text { Paraitinga }\end{array}$ & 527,87 & $99,01 \%$ \\
\hline $0^{\circ}$ & Silveiras & 785,90 & $65,50 \%$ & $5^{\circ}$ & Canas & 511,62 & $100 \%$ \\
\hline $1^{\circ}$ & $\begin{array}{l}\text { Campos do } \\
\text { Jordão }\end{array}$ & 751,87 & $98,86 \%$ & $6^{\circ}$ & Piquete & 479,25 & $100 \%$ \\
\hline $2^{\circ}$ & Caçapava & 743,45 & $100 \%$ & $7^{\circ}$ & Cunha & 453,63 & $64,65 \%$ \\
\hline $3^{\circ}$ & Bananal & 728,61 & $54,31 \%$ & $8^{\circ}$ & $\begin{array}{l}\text { Cachoeira } \\
\text { Paulista }\end{array}$ & 447,57 & $100 \%$ \\
\hline $4^{\circ}$ & Ubatuba & 721,62 & $100 \%$ & $9^{\circ}$ & Tremembé & 440,08 & $100 \%$ \\
\hline $5^{\circ}$ & Igaratá & 716,39 & $91,80 \%$ & $0^{\circ}$ & Aparecida & 395,22 & $96,68 \%$ \\
\hline
\end{tabular}

Fonte: Dados da pesquisa (2018).

Confirmando a pesquisa de Silva (2010), ao observar que maior eficiência não é necessariamente reflexo de maior investimento e corroborando com Oliveira (2012), pois alguns municípios que, apesar de realizarem maiores gastos, não atingiram a fronteira da 
eficiência calculada. A pesquisa demonstrou que embora tenham maiores investimentos não necessariamente esses municípios alcançaram a fronteira de eficiência técnica.

As cidades de Ilhabela e Jambeiro, com os investimentos per capita em $1^{\circ}$ e $4^{\circ}$ lugar, ficaram classificadas abaixo da fronteira de eficiência, com scores de 56,06\% e 49,27\% respectivamente. O nível de Jambeiro foi o pior da amostra estudada. Por outro lado, a cidade de Aparecida demonstrou o menor valor de investimento per capita em saúde e, no entanto, figurou entre os mais altos níveis, com score de eficiência de 96,68\%. Entre as 10 cidades com os maiores investimentos per capita, apenas 6 foram eficientes, do mesmo modo, entre as 10 com os menores investimentos per capita 6 foram eficientes.

Em sentido oposto, nas análises de Queiroz et al. (2013) evidenciou-se que os menores aportes de recursos se apresentaram menos eficientes. Os autores constataram que a conquista da eficiência nos indicadores de saúde pública municipal depende fortemente do montante de recursos.

\section{Considerações finais}

O objetivo desta pesquisa foi mensurar o nível de eficiência técnica dos municípios da Região Metropolitana do Vale do Paraíba e Litoral Norte de São Paulo, na aplicação dos recursos em saúde, no ano de 2014.

Para alcançar o objetivo pretendido, realizou-se uma pesquisa de natureza básica, com abordagem quantitativa, empregando-se a metodologia de Análise por Envoltória de Dados (DEA) com retornos variáveis de escala e orientação a produtos (output).

Os resultados demonstraram que $50 \%$ dos municípios analisados apresentaram eficiência técnica relativa de $100 \%$ no ano de 2014 e o índice médio alcançado na região foi de $87,7 \%$.

A pesquisa demonstrou que para uma cidade da amostra alcançar a fronteira de eficiência técnica não precisa necessariamente realizar maiores investimentos. Os dados obtidos revelaram que municípios com os maiores investimentos per capita figuram entre os piores resultados da $D E A$ e o oposto também foi verdadeiro, corroborando os estudos de Silva (2010) e Oliveira (2012). As cidades de Ilhabela e Jambeiro, com os investimentos per capita em $1^{\circ} \mathrm{e}$ $4^{\circ}$ lugar do ranking, ficaram classificadas abaixo da fronteira de eficiência, com escores de $56,06 \%$ e 49,27\%, respectivamente. Por outro lado, a cidade de Aparecida investiu o menor valor per capita em saúde e, no entanto, apresentou escore de eficiência de 96,68\%. 
O município de Canas foi identificado como benchmarks para 33,33\% das cidades ineficientes. Tremembé e Piquete também foram apontadas como benchmark para as cidades que não alcançaram a fronteira da eficiência, sendo $26,67 \%$ e $20 \%$, na devida ordem.

Ratificando o trabalho de Passoni (2014), verificou-se que a situação da saúde pública nos municípios estudados, examinada sob a ótica da eficiência relativa, sinaliza para melhorias. Contudo, alocar os recursos de maneira que o bem-estar alcançado seja o máximo possível é um desafio para os reguladores de políticas públicas. Dessa forma, a busca pelo aprimoramento constante é um instrumento para garantir a moralidade, impessoalidade, legalidade, publicidade, em suma a eficiência, que deve ser buscada com o intuito de nortear a gestão pública e o comportamento dos administradores públicos.

Os dados aqui demonstrados podem ser utilizados como estudo inicial do estudo da eficiência técnica dos municípios da Região Metropolitana do Vale do Paraíba e Litoral Norte de São Paulo, contudo, esta pesquisa encontrou algumas limitações, entre as quais o contorno bastante reduzido, por tratar estritamente do período de 2014, impedindo generalizações terminantes sobre o assunto. Outro fato é a atemporalidade dos dados disponíveis, que impede o estudo atualizado das variáveis. Além disso, a pesquisa se limitou a um estudo exploratório dos escores de eficiência das $D M U s$, de modo que não foram identificadas as causas relevantes da ineficiência técnica, ou variações ambientais e estruturais capazes de influenciar a destinação dos recursos públicos em saúde.

Isto posto, sugere-se para estudos futuros tanto a inclusão de outras variáveis, como a análise de outros períodos, com o propósito de abranger as possíveis interferências ambientais sobre a eficiência dos municípios analisados e permitir a comparação dos dados obtidos ao longo do tempo.

\section{Referências}

Andrade, M. M. (2010). Introdução à metodologia de trabalho científico: elaboração de trabalhos na graduação (10a ed.). São Paulo: Atlas.

Angulo-Meza, L., Biondi Neto, L., Mello, J. C. C. B. S. de, \& Gomes, E. G. (2005). ISYDS Integrated System for Decision Support (SIAD - Sistema Integrado de Apoio à Decisão): a software package for data envelopment analysis model. Pesquisa Operacional, 25(3), 493503.

Banker, R. D., Charnes, H., \& Cooper, W. W. (1984). Some models for estimating technical and scale inefficiencies in data envelopment analysis. Management Science, 30(9), 10781092. 
Charnes, A., Cooper, W., \& Rhodes, E. (1978). Measuring the efficiency of decision making units. European Journal of Operational Research, 2(6), 429-444.

Costa, F. L., \& Castanhar, J. C. (2003). Avaliação de programas públicos: desafios conceituais e metodológicos. Revista de Administração Pública, 37(5), 969-992.

Cozby, P. C. (2014). Métodos de Pesquisa em Ciências do Comportamento. São Paulo: Atlas.

Dalchiavon, E. C., \& Melo, C. O. (2016). Eficiência dos gastos públicos em educação, saúde e trabalho para o desenvolvimento dos municípios paranaenses. Gestão e Desenvolvimento em Revista, 2(2), 38-49.

Datasus (2014). Portal da Saúde TabNet. Recuperado em 25, maio, 2018, de http://www2.datasus.gov.br/DATASUS/index.php?area=02.

Dias, R. H. (2010). Eficiência da Atenção Primária à Saúde nos municípios Brasileiros. Dissertação de mestrado, Universidade de Brasília, Brasília, DF, Brasil. Disponível: http://repositorio.unb.br/handle/10482/8364.

Duarte, J. M., Diniz, J. A., Nobre, C. J., \& Araújo, R. J. (2016, julho). A Eficiência dos Gastos Públicos nos Serviços de Saúde Municipal. Anais do Congresso USP de Controladoria e Contabilidade, São Paulo, SP, Brasil, XVI.

Faria, F., Jannuzzi, P., \& Silva, S. (2008). Eficiência dos gastos municipais em saúde e educação: uma investigação através da análise envoltória no Estado do Rio de Janeiro. Revista de Administração Pública, 42(1), 155-177.

Farrel, M. (1957). The measurement of productive efficiency. Journal of the Royal Statistical Society, 120(3), 253-290.

Fonseca, P. C., \& Ferreira, M. A. (2009). Investigação dos níveis de eficiência na utilização de recursos no setor de saúde: uma análise das microrregiões de minas gerais. Saúde Social São Paulo - USP, 18(2), 199-213.

Gil, A. C. (2010). Como elaborar projetos de pesquisa (5a ed.). São Paulo: Atlas.

Grassetti, L., Gori, E., \& Bellio, R. (2003, giugno). Efficiency estimation of hospital services: a survey and multilevel developments. Atti del Convegno Intermedio "Analisi statistica multivariata per le scienze economico-sociali, le scienze naturali e la tecnologia”, Napoli, Italia. Retrieved 12, october, 2017, from http://www.dms.unina.it/sis2003/Lavori/vittadini/grassetti.pdf.

Hair Jr., J. F., Babin, B., Money, A. H., \& Samouel, P. (2005). Fundamentos de métodos de pesquisa em administração. Porto Alegre: Bookman.

Hartz, Z. M., \& Pouvourville, G. D. (1998, janeiro/junho). Avaliação dos Programas de Saúde: A Eficiência em Questão. Ciência \& Saúde Coletiva, 3(1), 68-82.

Machado Junior, S., Irffi, G., \& Benegas, M. (2011). Análise da Eficiência Técnica dos Gastos com Educação, Saúde e Assistência Social dos Municípios Cearenses. Planejamento e Políticas públicas, 1(36), 87-113. 
Malhotra, N. (2012). Pesquisa de Marketing: uma orientação aplicada (6a ed., L. B. Stefani, Trad.) Porto Alegre: Bookman.

Marinho, A. (2003). Avaliação da eficiência técnica nos serviços de saúde nos municípios do. Revista Brasileira de Economia, 57(3), 415-432.

Marinho, A., \& Façanha, L. O. (2001, junho). Hospitais universitários: avaliação comparativa de eficiência técnica. Texto para discussão, 805, 1-29.

Nunes, E. D., \& Sousa, E. P. (2016). Análise da eficiência no gerenciamento público com a saúde para os municípios cearenses. Anais do Encontro de Economia do Ceará em Debate, Fortaleza, CE, Brasil, XII. Recuperado em 10, dezembro, 2017, de http://www2.ipece.ce.gov.br/encontro/2016/trabalhos.pdf.

Passoni, P. F. M. (2014). Eficiência na oferta de serviços de saúde no Estado de São Paulo: uma análise comparativa entre municípios selecionados. Dissertação de mestrado, Universidade Federal de São Carlos, Sorocaba, SP, Brasil. Disponível: http://www.ppgec.ufscar.br/pesquisa/dissertacoes-1/dissertacoes2014/PedroFelipeMascarinPassonieconomia.pdf .

Queiroz, M. F., Silva, J. L., Figueiredo, J. S., \& Vale, F. F. (2013). Eficiência no gasto público com saúde: uma análise nos municípios do Rio Grande. Revista Econômica do Nordeste, 44(3), 761-776.

Schulz, S. J., Gollo, V., Rosa, F. S., \& Scarpin, J. E. (2014). Ranking das Unidades Federativas brasileiras frente ao seu desempenho na gestão de recursos da saúde. Revista de Gestão em Sistemas de Saúde, 3(2), 75-86.

Shaughnessy, J. J., Zechmeister, E. B., \& Zechmeister, J. S. (2012). Metodologia de pesquisa em psicologia (9a ed.). Porto Alegre: AMGH.

Silva, A. A. F.; Ferreira, M. C. O. A eficiência técnica nos investimentos no setor de saúde: um estudo na Região Metropolitana do Vale do Paraíba e Litoral Norte de São Paulo, Congresso Internacional de Administração, 2018.

Sistema de Informações sobre Orçamentos Públicos em Saúde - SIOPS (2014). Demonstrativo da despesa com saúde, conforme o anexo XVI do relatório resumido de execução orçamentária / RREO. Recuperado em 25 maio, 2018, de http://siops.datasus.gov.br/consleirespfiscal.php?S=1\&UF=35;\&Municipio=351050;\&Ano= $2014 \&$ Periodo $=20$

Tribunal de Contas do Estado de São Pop. (2017). IEG-M 2017: Índice do TCE mostra que eficiência das Prefeituras paulistas caiu. Recuperado em 10 outubro, 2017, de https://www4.tce.sp.gov.br/6524-ieg-m-2017-indice-tce-mostra-eficiencia-prefeituraspaulistas-caiu 